\title{
Ring of Adjacency Matrices of Digraphs
}

\author{
Epimaco A. Cabanlit, Jr.* \\ * Mathematics Department, Mindanao State University, General Santos City, Philippines
}

DOI: 10.29322/IJSRP.10.10.2020.p10616

http://dx.doi.org/10.29322/IJSRP.10.10.2020.p10616

\begin{abstract}
This paper shows, that under the piecewise addition modulo 2, and piecewise multiplication of adjacency matrices of digraphs, the basic properties for a ring are being satisfied.
\end{abstract}

Index Terms- Ring, Adjacency Matrices, Digraphs, Piecewise Addition modulo 2 and Piecewise Multiplication.

\section{INTRODUCTION}

The most general algebraic structure with two binary operations is the ring (Fraleigh, 1989).

The theory of rings grew out of the study of two particular classes of rings, polynomial rings in $\mathrm{n}$ variables over the real or complex numbers and the "integers" of an algebraic number field (Fraleigh, 1989).

There are many examples of rings which had been presented and studied. Here are some examples of rings: The rational, real and complex numbers form rings and they even form a field and likewise they are commutative rings. The Gaussian integers as do the Eisenstein integers. The set of integers modulo $\mathrm{n}$ forms also a ring. The power set of $\mathrm{S}$ becomes a a ring if we define addition to be the symmetric differences of sets and multiplication to be the intersection. This is an example of a Boolean ring. A Ring $R$ is said to be a Boolean ring if $a^{2}=a$ for every $a \in R$. The set of all continuous real-valued functions defined on the interval $[a, b]$ forms a ring (even an associative algebra) the operations are addition and multiplication of functions. We have also the ring of Cauchy Sequences. It is known that the set of all $n \times n$ matrices having elements of $R=$ set of real numbers as entries, under the usual addition and multiplication of matrices is a ring.

Cabanlit (2016) has shown that the set of $n \times n$ adjacency matrices of graphs under the piecewise addition and multiplication modulo 2 would constitute as a ring.

In this paper, we will show that the set of $n \times n$ adjacency matrices of digraphs under the piecewise addition and multiplication modulo 2 would constitute as a ring.

The adjacency matrix is the matrix associated with graphs and digraphs. Graphs and digraphs have many realistic applications. They are used as models in Sociology, Communication and Transportation.

\section{RESULTS AND DISCUSSION}

The adjacency matrix of a digraph that has $\mathrm{n}$ vertices is the $n \times n$ matrix whose $i, j t h$ element is 1 if there is at least one directed edge between $P_{i}$ and $P_{j}$ and zero otherwise, where $P_{i}$ and $P_{j}$ are vertices of a digraph. Note that the adjacency matrices in this paper are not necessarily symmetric matrices consisting of zeros and ones. Moreover, the diagonals are all zeros, since digraphs do not have loops. 
We consider the following definitions of sum and product of two adjacency matrices.

Definition 1. If $A=\left\lfloor a_{i j}\right\rfloor$ and $B=\left\lfloor b_{i j}\right\rfloor$ are $n \times n$ adjacency matrices of digraphs $G_{1}$ and $G_{2}$, then the sum $A \oplus B$ is the $n \times n$ adjacency matrix $C=\left\lfloor c_{i j}\right\rfloor$ of a new digraph $G_{3}$, defined by

$$
c_{i j}=a_{i j}+b_{i j}=\left\{\begin{array}{l}
0, \text { if } a_{i j}+b_{i j}=0 \text { or } a_{i j}+b_{i j}>1 \\
1, \text { if } a_{i j}+b_{i j}=1
\end{array} .\right.
$$

The matrix $\mathrm{C}$ is obtained by adding corresponding elements of $A$ and $B$ with the corresponding definition of the sum of values. This makes the sum of two adjacency matrices to be an adjacency matrix of another digraph.

Definition 2. If $A=\left\lfloor a_{i j}\right\rfloor$ and $B=\left\lfloor b_{i j}\right\rfloor$ are $n \times n$ adjacency matrices of digraphs $G_{1}$ and $G_{2}$, then the product $A \otimes B$ is the $n \times n$ adjacency matrix $C=\left\lfloor c_{i j}\right\rfloor$ of a new digraph $G_{3}$, defined by

$$
c_{i j}=a_{i j} b_{i j}=\left\{\begin{array}{l}
0, \text { if } a_{i j}=0 \text { or } b_{i j}=0, \text { or } a_{i j}=0 \text { and } b_{i j}=0 \\
1, \text { if } a_{i j}=1 \text { and } b_{i j}=1
\end{array} .\right.
$$

The matrix $\mathrm{C}$ is obtained by multiplying corresponding elements of $A$ and $B$ with the corresponding definition of the product. This makes the product of two adjacency matrices to be an adjacency matrix of another digraph.

With the above definitions of sum and product, we can now state that the set of $n \times n$ adjacency matrices together with these two binary operations constitute a ring.

Theorem 1. The set $R$ of $n \times n$ adjacency matrices of digraphs together with the sum $\oplus$ in Definition 1 and product $\otimes$ in Definition 2 is a ring.

Proof: To prove we must satisfy the eight (8) properties of a ring. Note that $R$ is a non empty set.

Property 1. The operation $\oplus$ is a binary operation.

From Definition 1, it is clear that the sum of two $n \times n$ adjacency matrices is also an adjacency matrix. Moreover, the diagonals are all zeros.

Property 2. The binary operation $\oplus$ is associative.

Let $A=\left\lfloor a_{i j}\right\rfloor, B=\left\lfloor b_{i j}\right\rfloor$ and $C=\left\lfloor c_{i j}\right\rfloor$ be $n \times n$ adjacency matrices of graphs $G_{1}, G_{2}$ and $G_{3}$, respectively. Then $A \oplus[B \oplus C]=[A \oplus B] \oplus C$. This follows from the fact that addition of $n \times n$ matrices is associative.

Property 3. The existence of an identity element $E$ in $R$ such that $E \oplus A=A \oplus E=A$ for all $A \in R$. (This element $E$ is an identity element for $\oplus$ on $R$ ).

The identity element in this case is the adjacency matrix whose entries are zeros.

That is $E=\left[\begin{array}{ccccc}0 & 0 & 0 & \cdots & 0 \\ 0 & 0 & 0 & \cdots & 0 \\ 0 & 0 & 0 & \cdots & 0 \\ \vdots & \vdots & \vdots & \ddots & \vdots \\ 0 & 0 & 0 & \cdots & 0\end{array}\right]$. 
Property 4. The existence of additive inverse.

The elements of the additive inverse of $A$, an $n \times n$ adjacency matrix are defined as follows: Suppose $a_{i j}$ are the elements of $A$, we let $-a_{i j}$ to be the corresponding elements of $-A$, where $-A$ is the additive inverse of $A$. Then, we have:

$$
-a_{i j}=\left\{\begin{array}{l}
0, \text { if } a_{i j}=0 \\
1, \text { if } a_{i j}=1
\end{array}\right. \text {. Thus, the inverse of A is A itself. }
$$

As an example: Suppose $A=\left[\begin{array}{lll}0 & 0 & 1 \\ 0 & 0 & 1 \\ 1 & 1 & 0\end{array}\right]$, then $-A=\left[\begin{array}{lll}0 & 0 & 1 \\ 0 & 0 & 1 \\ 1 & 1 & 0\end{array}\right]$. Thus, the inverse of A is A itself.

Property 5. The binary operation $\oplus$ is commutative.

The commutative law follows from the fact that addition of matrices is commutative.

Property 6. The operation $\otimes$ is a binary operation.

From Definition 2, it is clear that the product of two $n \times n$ adjacency matrices is also an adjacency matrix. Moreover, the entries are either zeros or ones and the diagonals are all zeros.

Property 7. The binary operation $\otimes$ is associative.

Let $A=\left\lfloor a_{i j}\right\rfloor, B=\left\lfloor b_{i j}\right\rfloor$ and $C=\left\lfloor c_{i j}\right\rfloor$, then we have

$$
\begin{aligned}
A \otimes(B \otimes C) & \left.=\left[a_{i j}\right\rfloor \otimes\left\{b_{i j}\right\rfloor \otimes\left\lfloor c_{i j}\right]\right\} \\
& =\left[a_{i j}\right] \otimes\left[b_{i j} c_{i j}\right] \\
& =\left[a_{i j}\left(b_{i j} c_{i j}\right)\right] \\
& =\left[\left(a_{i j} b_{i j}\right) c_{i j}\right] \\
& =\left[a_{i j} b_{i j}\right] \otimes\left[c_{i j}\right] \\
& =\left\{\left[a_{i j}\right] \otimes\left[b_{i j}\right]\right\} \otimes\left[c_{i j}\right] \\
& =(A \otimes B) \otimes C .
\end{aligned}
$$

Property 8. The Distributive Laws hold.

For all $A, B, C \in R$, we have

$A \otimes(B \oplus C)=(A \otimes B) \oplus(A \otimes C)$ and $(A \oplus B) \otimes C=(A \otimes B) \oplus(A \otimes C)$.

Let $A=\left\lfloor a_{i j}\right\rfloor, B=\left\lfloor b_{i j}\right\rfloor$ and $C=\left\lfloor c_{i j}\right\rfloor$, then we have 


$$
\begin{aligned}
A \otimes(B \oplus C) & \left.=\left\lfloor a_{i j}\right\rfloor \otimes\left\{b_{i j}\right\rfloor \oplus\left|c_{i j}\right|\right\} \\
& =\left[a_{i j}\right] \otimes\left[b_{i j}+c_{i j}\right] \\
& =\left[a_{i j}\left(b_{i j}+c_{i j}\right)\right] \\
& =\left[a_{i j} b_{i j}+a_{i j} c_{i j}\right] \\
& =\left[a_{i j} b_{i j}\right] \oplus\left[a_{i j} c_{i j}\right] \\
& =(A \otimes B) \oplus(A \otimes C) .
\end{aligned}
$$

The same way can be used to show that the right distributive law is true.

With the above definitions of a binary sum and product, and we have shown that all the properties are being satisfied, we are able to form a new ring consisting of all $n \times n$ adjacency matrices of a digraph.

\section{REFERENCES}

Cabanlit, E. A. Jr., (2016) Ring of Adjacency Matrices of Graphs. International Journal of Scientific and Research Publications. Volume 6, Issue 7, July 2016, pp.123126.

Fraleigh, John B. (1989). A first Course in Abstract Algebra. Addison-Wesley Publishing Company. USA.

Kemeny, John G., Snell, J. Laurie and Thompson, Gerald 1. (1978). Introduction to Finite Mathematics. $3^{\text {rd }}$ Edition. Prentice Hall of India, New Delhi, India.

Kolman, Bernard (1932). Introductory Linear Algebra with Applications. Macmillan Publishing Company, New York, USA.

Singh, Surjeet and Zameeruddin, Qazi (1990). Modern Algebra. Vikas Publishing House Pvt. Ltd., New Delhi.

Wright, Donald J. (1999). Introduction to Linear Algebra. McGraw-Hill Book Co., Singapore.

\section{AUTHOR}

First Author-Epimaco A. Cabanlit, Jr, Professor VI, Mathematics Department, Mindanao State University, General Santos City, Philippines. maco_727@yahoo.com. 